\title{
Domination in Functigraphs
}

\author{
Linda Eroh ${ }^{1}$ \\ Craig Larson ${ }^{4}$ \\ Ralucca Gera ${ }^{2}$ \\ ${ }^{1}$ Department of Mathematics, University of Wisconsin Oshkosh \\ Oshkosh, WI 54901; eroh@uwosh.edu \\ ${ }^{2}$ Department of Applied Mathematics, Naval Postgraduate School, \\ Monterey, CA 93943; rgera@nps.edu \\ ${ }^{3,5}$ Department of General Academics, Texas A\&M University at Galveston \\ Galveston, TX 77553; kangc@tamug.edu ${ }^{3}$, yie@tamug.edu ${ }^{5}$ \\ ${ }^{4}$ Department of Mathematics and Applied Mathematics, Virginia Commonwealth University \\ Richmond, VA 23284; clarson@vcu.edu
}

November 6, 2018

\begin{abstract}
Let $G_{1}$ and $G_{2}$ be disjoint copies of a graph $G$, and let $f: V\left(G_{1}\right) \rightarrow V\left(G_{2}\right)$ be a function. Then a functigraph $C(G, f)=(V, E)$ has the vertex set $V=V\left(G_{1}\right) \cup V\left(G_{2}\right)$ and the edge set $E=E\left(G_{1}\right) \cup E\left(G_{2}\right) \cup\left\{u v \mid u \in V\left(G_{1}\right), v \in V\left(G_{2}\right), v=f(u)\right\}$. A functigraph is a generalization of a permutation graph (also known as a generalized prism) in the sense of Chartrand and Harary. In this paper, we study domination in functigraphs. Let $\gamma(G)$ denote the domination number of $G$. It is readily seen that $\gamma(G) \leq \gamma(C(G, f)) \leq 2 \gamma(G)$. We investigate for graphs generally, and for cycles in great detail, the functions which achieve the upper and lower bounds, as well as the realization of the intermediate values.
\end{abstract}

Key Words: domination, permutation graphs, generalized prisms, functigraphs

2000 Mathematics Subject Classification: 05C69, 05C38

\section{Introduction and Definitions}

Throughout this paper, $G=(V(G), E(G))$ stands for a finite, undirected, simple and connected graph with order $|V(G)|$ and size $|E(G)|$. A set $D \subseteq V(G)$ is a dominating set of $G$ if for every vertex $v \in V(G) \backslash D$, there exists a vertex $u \in D$ such that $v$ and $u$ are adjacent. The domination number of a graph $G$, denoted by $\gamma(G)$, is the minimum of the cardinalities of all dominating sets of $G$. For earlier discussions on domination in graphs, see [3, 4, 10, 16]. For further reading on domination, refer to [13] and [14].

For any vertex $v \in V(G)$, the open neighborhood of $v$ in $G$, denoted by $N_{G}(v)$, is the set of all vertices adjacent to $v$ in $G$. The closed neighborhood of $v$, denoted by $N_{G}[v]$, is the 
set $N_{G}(v) \cup\{v\}$. Throughout the paper, we denote by $N(v)$ (resp., $N[v]$ ) the open (resp., closed) neighborhood of $v$ in $C(G, f)$. The maximum degree of $G$ is denoted by $\Delta(G)$. For a given graph $G$ and $S \subseteq V(G)$, we denote by $\langle S\rangle$ the subgraph induced by $S$. Refer to [8] for additional graph theory terminology.

Chartrand and Harary studied planar permutation graphs in [7. Hedetniemi introduced two graphs (not necessarily identical copies) with a function relation between them; he called the resulting object a "function graph" [15. Independently, Dörfler introduced a "mapping graph", which consists of two disjoint identical copies of a graph and additional edges between the two vertex sets specified by a function [11]. Later, an extension of permutation graphs, called functigraph, was rediscovered and studied in [9]. In the current paper, we study domination in functigraphs. We recall the definition of a functigraph in [9].

Definition 1.1. Let $G_{1}$ and $G_{2}$ be two disjoint copies of a graph $G$, and let $f$ be a function from $V\left(G_{1}\right)$ to $V\left(G_{2}\right)$. Then a functigraph $C(G, f)$ has the vertex set

$$
V(C(G, f))=V\left(G_{1}\right) \cup V\left(G_{2}\right),
$$

and the edge set

$$
E(C(G, f))=E\left(G_{1}\right) \cup E\left(G_{2}\right) \cup\left\{u v \mid u \in V\left(G_{1}\right), v \in V\left(G_{2}\right), v=f(u)\right\} .
$$

Throughout the paper, $V\left(G_{1}\right)$ denotes the domain of a function $f ; V\left(G_{2}\right)$ denotes the codomain of $f$; Range $(f)$ denotes the range of $f$. For a set $S \subseteq V\left(G_{2}\right)$, we denote by $f^{-1}(S)$ the set of all pre-images of the elements of $S$; i.e., $f^{-1}(S)=\left\{v \in V\left(G_{1}\right): f(v) \in S\right\}$. Also, $C_{n}$ denotes a cycle of length $n \geq 3$, and $i d$ denotes the identity function. Let $V\left(G_{1}\right)=\left\{u_{1}, u_{2}, \ldots, u_{n}\right\}$ and $V\left(G_{2}\right)=\left\{v_{1}, v_{2}, \ldots, v_{n}\right\}$. For simplicity, we sometimes refer to each vertex of the graph $G_{1}$ (resp., $G_{2}$ ) by the index $i$ (resp., $i^{\prime}$ ) of its label $u_{i}$ (resp., $v_{i}$ ) for $1 \leq i, i^{\prime} \leq n$. When $G=C_{n}$, we assume that the vertices of $G_{1}$ and $G_{2}$ are labeled cyclically. It is readily seen that $\gamma(G) \leq \gamma(C(G, f)) \leq 2 \gamma(G)$. We study the domination of $C\left(C_{n}, f\right)$ in great detail: for $n \equiv 0(\bmod 3)$, we characterize the domination number for an infinite class of functions and state conditions under which the upper bound is not achieved; for $n \equiv 1,2(\bmod 3)$, we prove that, for any function $f$, the domination number of $C\left(C_{n}, f\right)$ is strictly less than $2 \gamma\left(C_{n}\right)$. These results extend and generalize a result by Burger, Mynhardt, and Weakley in [6].

Domination number on permutation graphs (generalized prisms) has been extensively investigated in a great many articles, among these are [1, 2, 5, 6, 12]; the present paper primarily deepens - and secondarily broadens - the current state of knowledge.

\section{Domination Number of Functigraphs}

First we consider the lower and upper bounds of the domination number of $C(G, f)$.

Proposition 2.1. For any graph $G, \gamma(G) \leq \gamma(C(G, f)) \leq 2 \gamma(G)$. 
Proof. Let $D$ be a dominating set of $G$. Since a copy of $D$ in $G_{1}$ together with a copy of $D$ in $G_{2}$ form a dominating set of $C(G, f)$ for any function $f$, the upper bound follows. For the lower bound, assume there is a dominating set $D$ of $C(G, f)$ such that $|D|<\gamma(G)$. Let $D_{1}=D \cap V\left(G_{1}\right) \neq \emptyset$ and $D_{2}=D \cap V\left(G_{2}\right) \neq \emptyset$, with $D_{1} \cup D_{2}=D$. Now, for each $x \in D_{1}, x$ dominates exactly one vertex in $G_{2}$, namely $f(x)$. And so $D_{2} \cup\left\{f(x) \mid x \in D_{1}\right\}$ is a dominating set of $G_{2}$ of cardinality less than or equal to $|D|$, but $|D|<\gamma\left(G_{2}\right)$ - a contradiction.

Next we consider realization results for an arbitrary graph $G$.

Theorem 2.2. For any pair of integers $a, b$ such that $1 \leq a \leq b \leq 2 a$, there is a connected graph $G$ for which $\gamma(G)=a$ and $\gamma(C(G, f))=b$ for some function $f$.

Proof. Let the star $S_{i} \cong K_{1,4}$ have center $c_{i}$ for $1 \leq i \leq a$. Let $G$ be a chain of $a$ stars; i.e., the disjoint union of $a$ stars such that the centers are connected to form a path of length $a$ (and no other additional edges) - see Figure 2, Label the stars in the chain of the domain $G_{1}$ by $S_{1}, S_{2}, \ldots, S_{a}$ and label their centers by $c_{1}, c_{2}, \ldots, c_{a}$, respectively. Likewise, label the stars in the chain of the codomain $G_{2}$ by $S_{1}^{\prime}, S_{2}^{\prime}, \ldots, S_{a}^{\prime}$ and label their centers by $c_{1}^{\prime}, c_{2}^{\prime}, \ldots, c_{a}^{\prime}$, respectively. More generally, denote by $v^{\prime}$ the vertex in $G_{2}$ corresponding to an arbitrary $v$ in $G_{1}$.

We define $a+1$ functions from $G_{1}$ to $G_{2}$ as follows. Let $f_{0}$ be the "identity function"; i.e., $f_{0}(v)=v^{\prime}$. For each $i$ from 1 to $a$, let $f_{i}$ be the function which collapses $S_{1}$ through $S_{i}$ to $c_{1}^{\prime}$ through $c_{i}^{\prime}$, respectively, and which acts as the "identity" on the remaining vertices: $f_{i}\left(S_{j}\right)=c_{j}^{\prime}$ for $1 \leq j \leq i$ and $f_{i}(v)=v^{\prime}$ for $v \notin \bigcup_{1 \leq j \leq i} V\left(S_{j}\right)$. (See Figure 1.) Notice $\gamma(G)=a$.

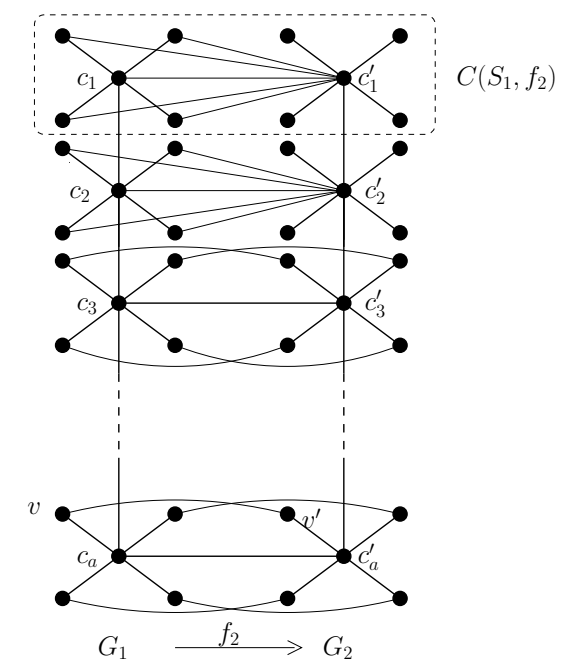

Figure 1: Realization Graphs

Claim: $\gamma\left(C\left(G, f_{i}\right)\right)=2 a-i$ for $0 \leq i \leq a$. 
First, $\gamma\left(C\left(G, f_{a}\right)\right)=a$ because $D_{a}=\left\{c_{1}^{\prime}, \ldots, c_{a}^{\prime}\right\}$ clearly dominates $C\left(G, f_{a}\right)$.

Second, consider $C\left(G, f_{0}\right) . D_{0}=\left\{c_{1}, \ldots, c_{a}, c_{1}^{\prime}, \ldots, c_{a}^{\prime}\right\}$, the set of centers in $G_{1}$ or $G_{2}$, is a dominating set; so $\gamma\left(C\left(G, f_{0}\right)\right) \leq 2 a$ as noted earlier. It suffices to show that $\gamma\left(C\left(G, f_{0}\right)\right) \geq$ $2 a$. It is clear that a dominating set $D$ consisting only of the centers must have size $2 a-$ for a pendant to be dominated, its neighboring center must be in $D$. We need to check that the replacement of centers by some (former) pendants (of $G_{1}$ or $G_{2}$ ) will only result in a dominating set $D^{\prime}$ such that $\left|D^{\prime}\right|>\left|D_{0}\right|$. It suffices to check $C\left(S_{i}, f_{0}\right)$ at each $i$, a subgraph of $C\left(G, f_{0}\right)$ - since pendant domination is a local question: the closed neighborhood of each pendant of $C\left(S_{i}, f_{0}\right)$ is contained within $C\left(S_{i}, f_{0}\right)$. It is easy to see that the unique minimum dominating set of $C\left(S_{i}, f_{0}\right)$ consists of the two centers $c_{i}$ and $c_{i}^{\prime}$.

Finally, the set $D_{i}=\left\{c_{i+1}, \ldots, c_{a}, c_{1}^{\prime}, \ldots, c_{a}^{\prime}\right\}$ is a minimum dominating set of $C\left(G, f_{i}\right)$ : In relation to $C\left(G, f_{0}\right)$, the subset $\left\{c_{1}, \ldots, c_{i}\right\}$ of $D_{0}$ is not needed since the set $\left\{c_{1}^{\prime}, \ldots, c_{i}^{\prime}\right\}$ dominates $\bigcup_{1 \leq j \leq i} V\left(S_{j}\right)$ in $C\left(G, f_{i}\right)$. The local nature of pendant domination and the fact that $\left.f_{i}\right|_{S_{j}}=\left.f_{0}\right|_{S_{j}}$ for $j>i$ ensure that $D_{i}$ has minimum cardinality.

\section{Characterization of Lower Bound}

We now present a characterization for $\gamma(C(G, f))=\gamma(G)$, in analogy with what was done for permutation-fixers in [5].

Theorem 3.1. Let $G_{1}$ and $G_{2}$ be two copies of a graph $G$ in $C(G, f)$. Then $\gamma(G)=$ $\gamma(C(G, f))$ if, and only if, there are sets $D_{1} \subseteq V\left(G_{1}\right)$ and $D_{2} \subseteq V\left(G_{2}\right)$ satisfying the following conditions:

1. $D_{1}$ dominates $V\left(G_{1}\right) \backslash f^{-1}\left(D_{2}\right)$,

2. $D_{2}$ dominates $V\left(G_{2}\right) \backslash f\left(D_{1}\right)$,

3. $D_{2} \cup f\left(D_{1}\right)$ is a minimum dominating set of $G_{2}$,

4. $\left|D_{1}\right|=\left|f\left(D_{1}\right)\right|$,

5. $D_{2} \cap f\left(D_{1}\right)=\emptyset$, and

6. $D_{1} \cap f^{-1}\left(D_{2}\right)=\emptyset$.

Proof. ( $\Longleftarrow)$ Suppose there are sets $D_{1} \subseteq V\left(G_{1}\right)$ and $D_{2} \subseteq V\left(G_{2}\right)$ satisfying the specified conditions. Clearly $D_{1} \cup D_{2}$ is a dominating set of $C(G, f)$. By assumption, $D_{2} \cup f\left(D_{1}\right)$ is a minimum dominating set of $G_{2}$. Since $\left|D_{1}\right|=\left|f\left(D_{1}\right)\right|$ and $D_{2} \cap f\left(D_{1}\right)=\emptyset, \gamma(G)=$ $\gamma\left(G_{2}\right)=\left|D_{2}\right|+\left|f\left(D_{1}\right)\right|=\left|D_{2}\right|+\left|D_{1}\right|$. Since $\gamma(G) \leq \gamma(C(G, f)) \leq\left|D_{1}\right|+\left|D_{2}\right|=\gamma(G)$, it follows that $\gamma(G)=\gamma(C(G, f))$.

$(\Longrightarrow)$ Let $D$ be any minimum dominating set of $C(G, f)$. Suppose then that $\gamma(G)=$ $\gamma(C(G, f))$ such that $D_{1}=D \cap V\left(G_{1}\right)$ and $D_{2}=D \cap V\left(G_{2}\right)$. So $\gamma(C(G, f))=\left|D_{1}\right|+\left|D_{2}\right|$. Note that the only vertices in $G_{2}$ that are dominated by $D_{1}$ are the vertices in $f\left(D_{1}\right)$ and the only vertices in $G_{1}$ that are dominated by $D_{2}$ are the vertices in $f^{-1}\left(D_{2}\right)$. Since $D$ is a 
dominating set of $C(G, f), D_{2}$ must dominate every vertex in $V\left(G_{2}\right) \backslash f\left(D_{1}\right)$, and $D_{1}$ must dominate every vertex in $V\left(G_{1}\right) \backslash f^{-1}\left(D_{2}\right)$.

Clearly $D_{2} \cup f\left(D_{1}\right)$ is a dominating set of $G_{2}$. Note that $\left|D_{1}\right| \geq\left|f\left(D_{1}\right)\right|$. So $\gamma(G)=$ $\gamma(C(G, f))=\left|D_{1}\right|+\left|D_{2}\right| \geq\left|D_{2}\right|+\left|f\left(D_{1}\right)\right| \geq \gamma\left(G_{2}\right)=\gamma(G)$. But then these terms must all be equal. In particular, $\left|D_{1}\right|=\left|f\left(D_{1}\right)\right|$ and $D_{2} \cup f\left(D_{1}\right)$ is a minimum dominating set of $G_{2}$. Furthermore, $D_{2} \cap f\left(D_{1}\right)=\emptyset$, else $D_{2} \cup f\left(D_{1}\right)$ is a dominating set of $G_{2}$ with fewer than $\gamma\left(G_{2}\right)$ vertices. Finally, suppose there is a vertex $v \in D_{1} \cap f^{-1}\left(D_{2}\right)$. So $v \in D_{1}$ and $v \in f^{-1}\left(D_{2}\right)$. But then $f(v) \in f\left(D_{1}\right)$ and $f(v) \in D_{2}$. But $f\left(D_{1}\right)$ and $D_{2}$ are disjoint. So, $D_{1} \cap f^{-1}\left(D_{2}\right)=\emptyset$.

It is known that for cycles $C_{n}(n \geq 3), \gamma\left(C_{n}\right)=\left\lceil\frac{n}{3}\right\rceil$. We now apply Theorem 3.1 to characterize the lower bound of $\gamma\left(C\left(C_{n}, f\right)\right)$.

Theorem 3.2. For the cycle $C_{n}(n \geq 3)$, let $G_{1}$ and $G_{2}$ be copies of $C_{n}$. Then $\gamma\left(C_{n}\right)=$ $\gamma\left(C\left(C_{n}, f\right)\right)$ if, and only if, there is a minimum dominating set $D=D_{1} \cup D_{2}$ of $C\left(C_{n}, f\right)$ such that either:

1. $D_{1}=\emptyset$ and $D_{2}$ is a minimum dominating set of $G_{2}$ and Range $(f) \subseteq D_{2}$, or

2. $n \equiv 1(\bmod 3), D_{2}$ is a minimum dominating set for $\left\langle V\left(G_{2}\right) \backslash\{v\}\right\rangle, D_{1}=\{w\}$, $f(w)=v$, and $f\left(V\left(G_{1}\right) \backslash N[w]\right) \subseteq D_{2}$.
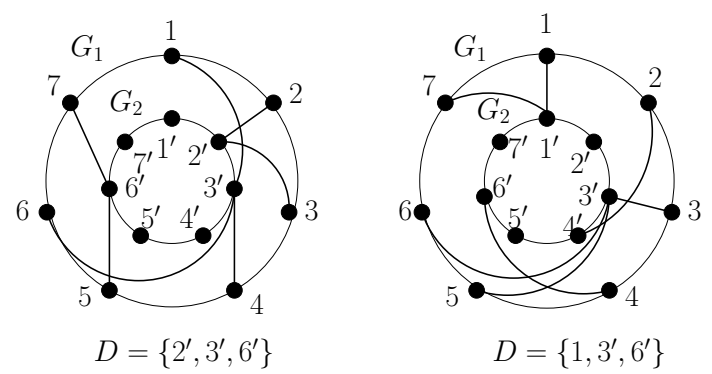

Figure 2: Examples of $\gamma\left(C\left(C_{n}, f\right)\right)=\gamma\left(C_{n}\right)$ for $n \equiv 1(\bmod 3)$

Proof. ( $\Longleftarrow)$ Suppose that there is a minimum dominating set $D$ of $C\left(C_{n}, f\right)$ satisfying the specified conditions. So $\gamma\left(C\left(C_{n}, f\right)\right)=|D|=\left|D_{1}\right|+\left|D_{2}\right|$. If $D_{2} \subseteq V\left(G_{2}\right)$ is a minimum dominating set of $C_{n}$ and Range $(f) \subseteq D_{2}$, then $D_{1}=\emptyset$. So $\gamma\left(C_{n}\right)=\left|D_{2}\right|=\left\lceil\frac{n}{3}\right\rceil$. Furthermore $\gamma\left(C\left(C_{n}, f\right)\right)=|D|=\left|D_{1}\right|+\left|D_{2}\right|=0+\gamma\left(G_{2}\right)$.

Suppose $n \equiv 1(\bmod 3), D_{2}$ dominates all but one vertex $v$ of $G_{2}, D_{1}=\{w\}, f(w)=v$, and $f\left(V\left(G_{1}\right) \backslash N[w]\right) \subseteq D_{2}$. Note that, since $n \equiv 1(\bmod 3), n=3 k+1$, for some positive integer $k$, and $\left\lceil\frac{n}{3}\right\rceil=k+1$. By assumption, $\gamma\left(C\left(C_{n}, f\right)\right)=|D|=\left|D_{1}\right|+\left|D_{2}\right|=1+\left|D_{2}\right|$. Since $\gamma\left(C_{n}\right)=k+1$, it remains to show that $\gamma\left(C\left(C_{n}, f\right)\right)=k+1$, which is equivalent to showing that $\left|D_{2}\right|=k$. Since $D_{2}$ is a minimum dominating set for $\left\langle V\left(G_{2}\right) \backslash\{v\}\right\rangle$ and $\left\langle V\left(G_{2}\right) \backslash\{v\}\right\rangle$ has domination number $k,\left|D_{2}\right|=k$. 
$(\Longrightarrow)$ Now suppose that $\gamma\left(C_{n}\right)=\gamma\left(C\left(C_{n}, f\right)\right)=\left\lceil\frac{n}{3}\right\rceil$. Let $D$ be a minimum dominating set satisfying the conditions of Theorem 3.1. There are three cases to consider: $n \equiv 0(\bmod 3)$, $n \equiv 1(\bmod 3)$, and $n \equiv 2(\bmod 3)$. In each case, Theorem 3.1 implies that $D_{2} \cup f\left(D_{1}\right)$ is a minimum dominating set of $G_{2}$ and $\left|D_{1}\right|=\left|f\left(D_{1}\right)\right|$. Since $f\left(D_{1}\right)$ must include all the vertices not dominated by $D_{2}$, it follows that $D$ must contain at least $\left|D_{2}\right|+\left(n-3\left|D_{2}\right|\right)=n-2\left|D_{2}\right|$ vertices.

If $n \equiv 0(\bmod 3)$, then $n=3 k$ for some positive integer $k$ and $\left\lceil\frac{n}{3}\right\rceil=k$. Note that $D_{2}$ dominates at most $3\left|D_{2}\right|$ vertices in $G_{2}$. There are at least $n-3\left|D_{2}\right|$ vertices in $G_{2}$ which are not dominated by $D_{2}$. If $\left|D_{2}\right|<k$ then $\gamma\left(C\left(C_{n}, f\right)\right)=|D| \geq n-2\left|D_{2}\right|>n-2 k=3 k-2 k=k$, contradicting the assumption that $\gamma\left(C\left(C_{n}, f\right)\right)=k$. So $\left|D_{2}\right|=k$. This implies $D_{1}=\emptyset$. And this, in turn, implies that $D_{2}$ must dominate all the vertices in $G_{1}$. So Range $(f) \subseteq D_{2}$.

In the remaining two cases, where $n \equiv 1$ or $n \equiv 2(\bmod 3)$, then $n=3 k+1$ or $n=3 k+2$, respectively, for some positive integer $k$ and $\gamma\left(C_{n}\right)=\left\lceil\frac{n}{3}\right\rceil=k+1$. From Theorem 3.1 it follows that $D_{2} \cup f\left(D_{1}\right)$ is a minimum dominating set of $G_{2}$. Since $D_{2}$ dominates at most $3\left|D_{2}\right|$ vertices in $G_{2}, D_{1}$ must dominate at least $n-3\left|D_{2}\right|$ vertices in $G_{2}$. If $\left|D_{2}\right|<k$, then $\gamma\left(C\left(C_{n}, f\right)\right)=|D| \geq n-2\left|D_{2}\right|>n-2 k=(3 k+1)-2 k=k+1$, contradicting the assumption that $\gamma\left(C\left(C_{n}, f\right)\right)=k+1$. So $\left|D_{2}\right| \geq k$. Since $|D|=k+1,\left|D_{2}\right| \leq k+1$. If $\left|D_{2}\right|=k+1$, then $D_{1}=\emptyset, f\left(D_{1}\right)=\emptyset$ and $D_{2} \cup f\left(D_{1}\right)=D_{2}$ is a minimum dominating set of $G_{2}$. Since $D$ is a dominating set of $C\left(C_{n}, f\right)$, it follows that $D_{2}$ must also dominate all the vertices in $D_{1}$ and, thus, Range $(f) \subseteq D_{2}$.

Let $n \equiv 1(\bmod 3)$. If $\left|D_{2}\right|=k$, then there is at least one vertex in $G_{2}$ not dominated by $D_{2}$. If there are $c>1$ vertices not dominated by $D_{2}$ then these vertices are a subset of $f\left(D_{1}\right)$ and Theorem 3.1 guarantees that $\left|D_{1}\right|=\left|f\left(D_{1}\right)\right| \geq c$ and, thus, $\gamma\left(C\left(C_{n}, f\right)\right) \geq k+c>k+1$, contradicting our assumption. So $c=1$. There is only one vertex $v \in V\left(G_{2}\right)$ which is not dominated by $D_{2}$. $D_{1}$ can only contain a single vertex $w$ (or $|D|$ will again be too large) and $f(w)=v$. Since $w$ dominates $N[w]$ in $G_{1}$, it follows that $D_{2}$ must dominate $V\left(G_{1}\right) \backslash N[w]$. So $f\left(V\left(G_{1}\right) \backslash N[w]\right) \subseteq D_{2}$.

Let $n \equiv 2(\bmod 3)$. If $\left|D_{2}\right|=k$, then there are at least two vertices in $G_{2}$ not dominated by $D_{2}$. But then these vertices must be a subset of $f\left(D_{1}\right)$ and $\left|f\left(D_{1}\right)\right| \geq 2$. Since $\left|D_{1}\right|=$ $\left|f\left(D_{1}\right)\right|,\left|D_{1}\right| \geq 2$. But then $k+1=\gamma(C(G, f))=|D|=\left|D_{1}\right|+\left|D_{2}\right| \geq 2+k$, which is a contradiction. So $\left|D_{2}\right|=k+1$.

Next we consider the domination number of $C\left(C_{3}, f\right)$.

Lemma 3.3. Let $G_{1}$ and $G_{2}$ be two copies of $C_{3}$. Then $\gamma\left(C\left(C_{3}, f\right)\right)=2 \gamma\left(C_{3}\right)$ if and only if $f$ is not a constant function.

Proof. $(\Longleftarrow)$ Suppose that $f$ is not a constant function. Then, for each vertex $v \in V\left(C\left(C_{3}, f\right)\right)$, $\operatorname{deg}(v) \leq 4$ and hence $N[v] \varsubsetneqq V\left(C\left(C_{3}, f\right)\right)$. Thus $\gamma\left(C\left(C_{3}, f\right)\right) \geq 2$. Since there exists a dominating set consisting of one vertex from each of $G_{1}$ and $G_{2}, \gamma\left(C\left(C_{3}, f\right)\right)=2$.

$(\Longrightarrow)$ Suppose that $f$ is a constant function, say $f(w)=a$ for some $a \in V\left(G_{2}\right)$ and for all $w \in V\left(G_{1}\right)$. Then $N[a]=V\left(C\left(C_{3}, f\right)\right)$, and thus $\gamma\left(C\left(C_{3}, f\right)\right)=1=\gamma\left(C_{3}\right)$. 
As an immediate consequence of Theorem 3.2 and Lemma 3.3, we have the following.

Corollary 3.4. There is no permutation $f$ such that $\gamma\left(C\left(C_{n}, f\right)\right)=\gamma\left(C_{n}\right)$ for $n=3$ or $n \geq 5$.

Now we consider $C(G, f)$ when $G=C_{n}(n \geq 3)$ and $f$ is the identity function.

Theorem 3.5. Let $G_{1}$ and $G_{2}$ be two copies of the cycle $C_{n}$ for $n \geq 3$. Then

$$
\gamma\left(C\left(C_{n}, i d\right)\right)=\left\{\begin{array}{lll}
\left\lceil\frac{n}{2}\right\rceil & \text { if } n \not \equiv 2 \quad(\bmod 4) \\
\frac{n}{2}+1 & \text { if } n \equiv 2 \quad(\bmod 4)
\end{array}\right.
$$

Proof. Since $C\left(C_{n}, i d\right)$ is 3 -regular, each vertex in $C\left(C_{n}, i d\right)$ can dominate 4 vertices. We consider four cases.

Case 1. $n=4 k$ : Since $\left|V\left(C\left(C_{n}, i d\right)\right)\right|=8 k$, we have $\gamma\left(C\left(C_{n}, i d\right)\right) \geq\left\lceil\frac{8 k}{4}\right\rceil=2 k$. Since $\cup_{j=0}^{k-1}\left\{4 j+1,(4 j+3)^{\prime}\right\}$ is a dominating set of $C\left(C_{n}, i d\right)$ with cardinality $2 k$, we conclude that $\gamma\left(C\left(C_{n}, i d\right)\right)=2 k=\left\lceil\frac{n}{2}\right\rceil$.

Case 2. $n=4 k+1$ : Since $\left|V\left(C\left(C_{n}, i d\right)\right)\right|=2(4 k+1)=8 k+2$, we have $\gamma\left(C\left(C_{n}, i d\right)\right) \geq$ $\left\lceil\frac{8 k+2}{4}\right\rceil=2 k+1$. Since $\left(\cup_{j=0}^{k}\{4 j+1\}\right) \bigcup\left(\cup_{i=0}^{k-1}\left\{(4 i+3)^{\prime}\right\}\right)$ is a dominating set of $C\left(C_{n}, i d\right)$ with cardinality $2 k+1$, we have $\gamma\left(C\left(C_{n}, i d\right)\right)=2 k+1=\left\lceil\frac{n}{2}\right\rceil$.

Case 3. $n=4 k+2$ : Notice that $\left(\cup_{j=0}^{k}\{4 j+1\}\right) \bigcup\left(\cup_{i=0}^{k-1}\left\{(4 i+3)^{\prime}\right\}\right) \bigcup\left\{(4 k+2)^{\prime}\right\}$ is a dominating set of $C\left(C_{n}, i d\right)$ with cardinality $2 k+2=\frac{n}{2}+1$; thus $\gamma\left(C\left(C_{n}, i d\right)\right) \leq 2 k+2$. Since $\left|V\left(C\left(C_{n}, i d\right)\right)\right|=2(4 k+2)=8 k+4, \gamma\left(C\left(C_{n}, i d\right)\right) \geq\left\lceil\frac{8 k+4}{4}\right\rceil=2 k+1$; indeed, $\gamma\left(C\left(C_{n}, i d\right)\right)=2 k+1$ only if every vertex is dominated by exactly one vertex of a dominating set; i.e., no double domination is allowed. However, we show that there must exist a doubly-dominated vertex for any dominating set by the following descent argument: Let the graph $A_{0}$ be $P_{4 k+3} \times K_{2}$ where the bottom row is labeled $1,2, \ldots, 4 k+2,1$ and the top row is labeled $1^{\prime}, 2^{\prime}, \ldots,(4 k+2)^{\prime}, 1^{\prime}$; note that $C\left(C_{n}, i d\right)$ is obtained by identifying the two end-edges each with end-vertices labeled 1 and $1^{\prime}$. Without loss of generality, choose $1^{\prime}$ to be in a dominating set $D$. For each vertex to be singly dominated, we delete vertices $1^{\prime}(s), 1(s), 2^{\prime}$, and $(4 k+2)^{\prime}$, as well as their incident edges, to obtain a derived graph $A_{1}$. In $A_{1}$, vertices 2 and $4 k+2$ are end-vertices and neither may belong to $D$ as each only dominates two vertices in $A_{1}$. This forces support vertices 3 and $4 k+1$ in $A_{1}$ to be in $D$. Deleting vertices $2,3,3^{\prime}, 4,4 k+2,4 k+1,(4 k+1)^{\prime}$, and $4 k$ and incident edges results in the second derived graph $A_{2}$. After $k$ iterations, $A_{k}$ is the extension of $P_{3} \times P_{2}$ by two leaves at both ends of either the top or the bottom row (see Figure 3); $A_{k}$, which has eight vertices, clearly requires three vertices to be dominated. Thus, we conclude that $\gamma\left(C\left(C_{n}, i d\right)\right)=2 k+2=\frac{n}{2}+1$.

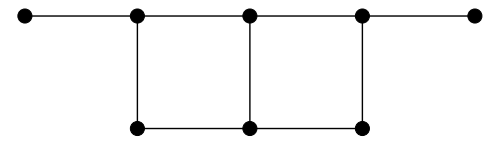

Figure 3: $A_{k}$ in the $n=4 k+2$ case 
Case 4. $n=4 k+3$ : Since $\left|V\left(C\left(C_{n}, i d\right)\right)\right|=2(4 k+3)=8 k+6$, we have $\gamma\left(C\left(C_{n}, i d\right)\right) \geq$ $\left\lceil\frac{8 k+6}{4}\right\rceil=2 k+2$. Since $\cup_{j=0}^{k}\left\{4 j+1,(4 j+3)^{\prime}\right\}$ is a dominating set of $C\left(C_{n}, i d\right)$ with cardinality $2 k+2$, we conclude that $\gamma\left(C\left(C_{n}, i d\right)=2 k+2=\left\lceil\frac{n}{2}\right\rceil\right.$.

As a consequence of Theorem 3.5, we have the following result.

Corollary 3.6. 1. $\gamma\left(C\left(C_{n}, i d\right)\right)=\gamma\left(C_{n}\right)$ if and only if $n=4$.

2. $\gamma\left(C\left(C_{n}, i d\right)\right)=2 \gamma\left(C_{n}\right)$ if and only if $n=3$ or $n=6$.

By Corollary 3.4 and Theorem 3.5, we have the following result.

Proposition 3.7. For a permutation $f, \gamma\left(C\left(C_{n}, f\right)\right)=\gamma\left(C_{n}\right)$ if and only if $C\left(C_{n}, f\right) \cong$ $C\left(C_{4}, i d\right)$.

Proof. $(\Longleftarrow)$ If $C\left(C_{4}, f\right) \cong C\left(C_{4}, i d\right)$, then $\gamma\left(C_{4}\right)=2=\gamma\left(C\left(C_{4}, i d\right)\right)$ by Theorem 3.5 .

$(\Longrightarrow)$ Let $\gamma\left(C\left(C_{n}, f\right)\right)=\gamma\left(C_{n}\right)$ for $n \geq 3$. By Corollary [3.4, $n=4$. If $f$ is a permutation, then $C\left(C_{4}, f\right)$ is isomorphic to the graph (A) or (B) in Figure 4 (refer to [7, 9] for details). If $C\left(C_{4}, f\right) \cong C\left(C_{4}, i d\right)$, then we are done. If $C\left(C_{4}, f\right)$ is as in (B) of Figure 4, we claim

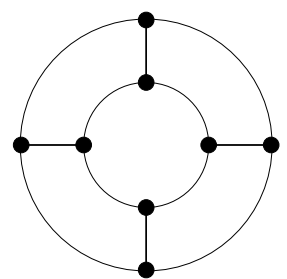

(A)

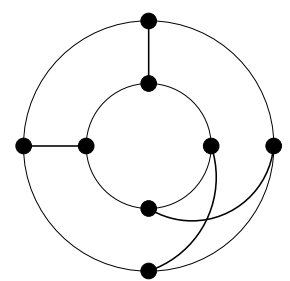

(B)

Figure 4: Two non-isomorphic graphs of $C\left(C_{4}, f\right)$ for a permutation $f$

that $\gamma\left(C\left(C_{4}, f\right)\right) \geq 3$.

Since $\left|V\left(C\left(C_{4}, f\right)\right)\right|=8$ and $C\left(C_{4}, f\right)$ is 3-regular, $D=\left\{w_{1}, w_{2}\right\}$ dominates $C\left(C_{4}, f\right)$ only if no vertex in $C\left(C_{4}, f\right)$ is dominated by both $w_{1}$ and $w_{2}$. It suffices to consider two cases, using the fact that $C\left(C_{4}, f\right) \cong C\left(C_{4}, f^{-1}\right)$.

(i) $D=\left\{w_{1}, w_{2}\right\} \subseteq V\left(G_{1}\right)$,

(ii) $w_{1} \in V\left(G_{1}\right)$ and $w_{2} \in V\left(G_{2}\right)$.

Also, we only need to consider $w_{1}$ and $w_{2}$ such that $w_{1} w_{2} \notin E\left(C\left(C_{4}, f\right)\right)$. By symmetry, there is only one specific case to check in case (i). In case (ii), by fixing a vertex in $V\left(G_{1}\right)$, we see that there are three cases to check. In each case, for any $D=\left\{w_{1}, w_{2}\right\}$, $N\left[w_{1}\right] \cap N\left[w_{2}\right] \neq \emptyset$. Thus $\gamma\left(C\left(C_{4}, f\right)\right)>2$.

\section{Upper Bound of $\gamma\left(C\left(C_{n}, f\right)\right)$}

In this section we investigate domination number of functigraphs for cycles: We show that $\gamma\left(C\left(C_{n}, f\right)\right)<2 \gamma\left(C_{n}\right)$ for $n \equiv 1,2(\bmod 3)$. For $n \equiv 0(\bmod 3)$, we characterize 
the domination number for an infinite class of functions and state conditions under which the upper bound is not achieved. Our result in this section generalizes a result of Burger, Mynhardt, and Weakley in [6] which states that no cycle other than $C_{3}$ and $C_{6}$ is a universal doubler (i.e., only for $n=3,6, \gamma\left(C\left(C_{n}, f\right)\right)=2 \gamma\left(C_{n}\right)$ for any permutation $f$ ).

\subsection{A characterization of $\gamma\left(C\left(C_{3 k+1}, f\right)\right)$}

Proposition 4.1. For any function $f, \gamma\left(C\left(C_{3 k+1}, f\right)\right)<2 \gamma\left(C_{3 k+1}\right)$ for $k \in \mathbb{Z}^{+}$.

Proof. Without loss of generality, we may assume that $u_{1} v_{1} \in E\left(C\left(C_{n}, f\right)\right)$. Since $D=$ $\left\{v_{1}\right\} \cup\left\{u_{3 j}, v_{3 j} \mid 1 \leq j \leq k\right\}$ is a dominating set of $C\left(C_{3 k+1}, f\right)$ with $|D|=2 k+1$ for any function $f, \gamma\left(C\left(C_{3 k+1}, f\right)\right)<2 \gamma\left(C_{3 k+1}\right)$ for $k \in \mathbb{Z}^{+}$.

\subsection{A characterization of $\gamma\left(C\left(C_{3 k+2}, f\right)\right)$}

We begin with the following example showing $\gamma\left(C\left(C_{5}, f\right)\right)<2 \gamma\left(C_{5}\right)$ for any function $f$.

Example 4.2. For any function $f, \gamma\left(C\left(C_{5}, f\right)\right)<2 \gamma\left(C_{5}\right)$.

Proof. Let $G=C_{5}, V\left(G_{1}\right)=\{1,2,3,4,5\}$, and $V\left(G_{2}\right)=\left\{1^{\prime}, 2^{\prime}, 3^{\prime}, 4^{\prime}, 5^{\prime}\right\}$. If $\mid$ Range $(f) \mid \leq 2$, we can choose a dominating set consisting of all vertices in the range and, if necessary, an additional vertex. If $\mid$ Range $(f) \mid=3$, then we can choose the range as a dominating set.

So, let $\mid$ Range $(f) \mid \geq 4$. Then $f$ is bijective on at least three vertices in the domain and their image. By the pigeonhole principle, there exist two adjacent vertices, say 1 and 2, on which $f$ is bijective. Let $f(1)=1^{\prime}$. Then, by relabeling if necessary, $f(2)=2^{\prime}$ or $f(2)=3^{\prime}$. Suppose $f(2)=3^{\prime}$. Then $D=\left\{1^{\prime}, 3^{\prime}, 4\right\}$ forms a dominating set, and we are done. Suppose then $f(2)=2^{\prime}$. We consider two cases.

Case 1. $\mid$ Range $(f) \mid=4$ : By symmetry, $5^{\prime} \notin \operatorname{Range}(f)$ is the same as $3^{\prime} \notin \operatorname{Range}(f)$. So, consider two distinct cases, $5^{\prime} \notin \operatorname{Range}(f)$ and $4^{\prime} \notin \operatorname{Range}(f)$. If $5^{\prime} \notin \operatorname{Range}(f)$, then $D=\left\{1,3^{\prime}, 4^{\prime}\right\}$ forms a dominating set. If $4^{\prime} \notin \operatorname{Range}(f)$, then $D=\left\{1,3^{\prime}, 5^{\prime}\right\}$ forms a dominating set. In either case, we have $\gamma\left(C\left(C_{5}, f\right)\right)<2 \gamma\left(C_{5}\right)$.

Case 2. $f$ is a bijection (permutation): Recall $f(1)=1^{\prime}$ and $f(2)=2^{\prime}$; there are thus $3 !=6$ permutations to consider. Using the standard cycle notation, the permutations are $(3,4)$, $(3,5),(4,5),(3,4,5),(3,5,4)$, and identity. However, they induce only four non-isomorphic graphs, since $(3,4)$ and $(4,5)$ induce isomorphic graphs and $(3,4,5)$ and $(3,5,4)$ induce isomorphic graphs. If $f$ is either $(3,4)$ or $(3,4,5)$, then $D=\left\{2,3^{\prime}, 5^{\prime}\right\}$ is a dominating set. If $f$ is $(3,5)$, then $D=\left\{1^{\prime}, 3,3^{\prime}\right\}$ is a dominating set. When $f$ is the identify function, $D=\left\{1^{\prime}, 3,5^{\prime}\right\}$ is a dominating set. It is thus verified that $\gamma\left(C\left(C_{5}, f\right)\right)<2 \gamma\left(C_{5}\right)$.

Remark 4.3. Example 4.2 has the following implication. Given $C\left(C_{3 k+2}, f\right)$ for $k \in \mathbb{Z}^{+}$, suppose there exist five consecutive vertices being mapped by $f$ into five consecutive vertices. Then $\gamma\left(C\left(C_{3 k+2}, f\right)\right)<2 \gamma\left(C_{3 k+2}\right)=2 k+2$, and here is a proof. Relabeling if necessary, we may assume that $\left\{u_{1}, u_{2}, u_{3}, u_{4}, u_{5}\right\}$ are mapped into $\left\{v_{1}, v_{2}, v_{3}, v_{4}, v_{5}\right\}$; let $S=\left\{u_{i}, v_{i} \mid\right.$ $1 \leq i \leq 5\}$. Then $\langle S\rangle$ in $C\left(C_{3 k+2}, f\right)$ and the additional edge set $\left\{u_{1} u_{5}, v_{1} v_{5}\right\}$ form a graph 
isomorphic to a $C\left(C_{5}, f\right)$, which has a dominating set $S_{0}$ with $\left|S_{0}\right| \leq 3$. In $C\left(C_{3 k+2}, f\right)$, if $S$ is dominated by $S_{0}$, then $D=S_{0} \cup\left\{u_{3 j+1} \mid 2 \leq j \leq k\right\} \cup\left\{v_{3 j+1} \mid 2 \leq j \leq k\right\}$ forms a dominating set for $C\left(C_{3 k+2}, f\right)$ with at most $2 k+1$ vertices. If $u_{1}$ is not dominated by $S_{0}$ in $C\left(C_{3 k+2}, f\right)$, then it is dominated solely by $u_{5}$ of $S_{0}$ in $C\left(C_{5}, f\right)$. But then $u_{6}$ is dominated by $u_{5}$ in $C\left(C_{3 k+2}, f\right)$ and we can replace $\left\{u_{3 j+1} \mid 2 \leq j \leq k\right\}$ with $\left\{u_{3 j+2} \mid 2 \leq j \leq k\right\}$ to form D. Similarly, if $u_{5}$ is not dominated by $S_{0}$ in $C\left(C_{3 k+2}, f\right)$, then it is dominated solely by $u_{1}$ of $S_{0}$ in $C\left(C_{5}, f\right)$. Then $u_{3 k+2}$ is dominated by $u_{1}$ in $C\left(C_{3 k+2}, f\right)$ and we can replace $\left\{u_{3 j+1} \mid 2 \leq j \leq k\right\}$ with $\left\{u_{3 j} \mid 2 \leq j \leq k\right\}$ to form $D$. The cases where $v_{1}$ or $v_{5}$ is not dominated by $S_{0}$ in $C\left(C_{3 k+2}, f\right)$ can be likewise handled. Thus, if five consecutive vertices are mapped by $f$ into five consecutive vertices, then $\gamma\left(C\left(C_{3 k+2}, f\right)\right) \leq 2 k+1<2 k+2=$ $2 \gamma\left(C_{3 k+2}\right)$.

Remark 4.4. Unlike $C\left(C_{5}, f\right)$, it is easily checked that $\gamma\left(C\left(P_{5}, f\right)\right)=2 \gamma\left(P_{5}\right)$ for the function $f$ given in Figure 5 , where $P_{5}$ is the path on five vertices.

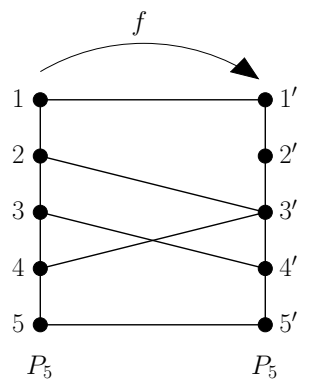

Figure 5: An example where $\gamma\left(C\left(P_{5}, f\right)\right)=2 \gamma\left(P_{5}\right)$

Now we consider the domination number of $C\left(C_{3 k+2}, f\right)$ for a non-permutation function $f$, where $k \in \mathbb{Z}^{+}$.

Theorem 4.5. Let $f: V\left(C_{3 k+2}\right) \rightarrow V\left(C_{3 k+2}\right)$ be a function which is not a permutation. Then $\gamma\left(C\left(C_{3 k+2}, f\right)\right)<2 \gamma\left(C_{3 k+2}\right)=2 k+2$.

Proof. Suppose $f$ is a function from $C_{3 k+2}$ to $C_{3 k+2}$ and $f$ is not a permutation. There must be a vertex $v_{1}$ in $G_{2}$ such that $\operatorname{deg}\left(v_{1}\right) \geq 4$ in $C\left(C_{3 k+2}, f\right)$. Define the sets $V_{1}=\left\{v_{3 i+1} \mid 0 \leq\right.$ $i \leq k\}, V_{2}=\left\{v_{3 i+2} \mid 0 \leq i \leq k\right\}$, and $V_{3}=\left\{v_{3 i} \mid 1 \leq i \leq k\right\} \cup\left\{v_{1}\right\}$. Notice that each of these three sets is a minimum dominating set of $G_{2}$ of cardinality $k+1$. Also, notice that $\left|f^{-1}\left(V_{1}\right)\right|+\left|f^{-1}\left(V_{2}\right)\right|+\left|f^{-1}\left(V_{3}\right)\right|$ counts every vertex in the pre-image of $V\left(G_{2}\right) \backslash\left\{v_{1}\right\}$ once and every vertex in the pre-image of $\left\{v_{1}\right\}$ twice, so $\left|f^{-1}\left(V_{1}\right)\right|+\left|f^{-1}\left(V_{2}\right)\right|+\left|f^{-1}\left(V_{3}\right)\right| \geq 3 k+4$. By the Pigeonhole Principle, $\left|f^{-1}\left(V_{i}\right)\right| \geq\left\lceil\frac{3 k+4}{3}\right\rceil=k+2$ for some $i$. Set $D_{2}=V_{i}$ for this $i$ and notice that $D_{2}$ is a dominating set of $G_{2}$ with cardinality $k+1$ and $\left|f^{-1}\left(D_{2}\right)\right| \geq k+2$.

Without loss of generality, we may assume that $u_{1}$ is in $f^{-1}\left(D_{2}\right)$. If there exists $0 \leq i \leq k$ such that $u_{3 i+2}$ is also in the pre-image of $D_{2}$, then $D_{1}=\left\{u_{3 j} \mid 1 \leq j \leq i\right\} \cup\left\{u_{3 j+1} \mid i+1 \leq\right.$ $j \leq k\}$ dominates the remaining vertices of $G_{1}$. Otherwise, there are at least $k+1$ vertices in $f^{-1}\left(D_{2}\right) \cap\left\{u_{3 j}, u_{3 j+1} \mid 1 \leq j \leq k\right\}$. By the Pigeonhole Principle, there exist two vertices $u_{3 j_{0}}$ and $u_{3 j_{0}+1}$ in $f^{-1}\left(D_{2}\right)$ which are adjacent in $G_{1}$. Then $D_{1}=\left\{u_{1}\right\} \cup\left\{u_{3 j+1} \mid 1 \leq j \leq\right.$ 
$\left.j_{0}-1\right\} \cup\left\{u_{3 j^{\prime}} \mid j_{0}+1 \leq j^{\prime} \leq k\right\}$ dominates the remaining vertices of $G_{1}$. In either case, $D_{1} \cup D_{2}$ is a dominating set of $C\left(C_{3 k+2}, f\right)$ with $2 k+1$ vertices.

For $G_{i} \subseteq C(G, f)(i=1,2)$, the distance between $x$ and $y$ in $\left\langle V\left(G_{i}\right)\right\rangle$ is denoted by $d_{G_{i}}(x, y)$.

Theorem 4.6. Let $f: V\left(C_{3 k+2}\right) \rightarrow V\left(C_{3 k+2}\right)$ be a function, where $k \in \mathbb{Z}^{+}$. For the cycle $C_{3 k+2}$, if there exist two vertices $x$ and $y$ in $G_{1}$ such that $d_{G_{1}}(x, y) \equiv 1(\bmod 3)$ and $d_{G_{2}}(f(x), f(y)) \not \equiv 1(\bmod 3)$, then $\gamma\left(C\left(C_{3 k+2}, f\right)\right)<2 \gamma\left(C_{3 k+2}\right)$.

Proof. Let $x=1$ and $y=3 a+2$ for a nonnegative integer $a$. By relabeling, if necessary, we may assume that $f(x)=1^{\prime}$. Note that $D_{1}=\left(\cup_{i=1}^{a}\{3 i\}\right) \cup\left(\cup_{i=a+1}^{k}\{3 i+1\}\right)$ dominates vertices in $V\left(G_{1}\right) \backslash\{x, y\}$. If $f(x)=1^{\prime}=f(y)$, let $D_{2}$ be any minimum dominating set of $G_{2}$ containing $1^{\prime}$. Then $D=D_{1} \cup D_{2}$ is a dominating set of $C\left(C_{3 k+2}, f\right)$ with $|D| \leq 2 k+1$. Thus, we assume that $f(x) \neq f(y)$. Since $d_{G_{2}}(f(x), f(y)) \not \equiv 1(\bmod 3), f(y)=(3 \ell)^{\prime}$ or $f(y)=(3 \ell+1)^{\prime}$ for some $\ell(1 \leq \ell \leq k)$. First, consider when $\ell>1$. If $f(y)=(3 \ell)^{\prime}$, let $D_{2}=\left(\cup_{i=1}^{\ell-1}\left\{(3 i+1)^{\prime}\right\}\right) \cup\left(\cup_{i=\ell+1}^{k}\left\{(3 i)^{\prime}\right\}\right) \cup\left\{1^{\prime},(3 \ell)^{\prime}\right\}$; and if $f(y)=(3 \ell+1)^{\prime}$, let $D_{2}=$ $\left(\cup_{i=1}^{\ell-1}\left\{(3 i+1)^{\prime}\right\}\right) \cup\left(\cup_{i=\ell+1}^{k}\left\{(3 i+1)^{\prime}\right\}\right) \cup\left\{1^{\prime},(3 \ell+1)^{\prime}\right\}$. Second, consider when $\ell=1$. If $f(y)=(3 \ell)^{\prime}$, let $D_{2}=\left(\cup_{i=1}^{k}\left\{(3 i)^{\prime}\right\}\right) \cup\left\{1^{\prime}\right\}$; if $f(y)=(3 \ell+1)^{\prime}$, let $D_{2}=\left(\cup_{i=1}^{k}\left\{(3 i+1)^{\prime}\right\}\right) \cup\left\{1^{\prime}\right\}$. Notice that $D_{2}$ dominates $V\left(G_{2}\right) \cup\{x, y\}$ in each case. Thus $D=D_{1} \cup D_{2}$ is a dominating set of $C\left(C_{3 k+2}, f\right)$ with $|D|=\left|D_{1}\right|+\left|D_{2}\right|=k+k+1=2 k+1<2 \gamma\left(C_{3 k+2}\right)=2 k+2$.

Next we consider $C\left(C_{3 k+2}, f\right)$ for a permutation $f$.

Lemma 4.7. Let $f$ be a monotone increasing function from $S=\{1,2, \ldots, n\}$ to $\mathbb{Z}$ such that $f(1)=1$. If $|j-i| \equiv 1(\bmod 3)$ implies $|f(j)-f(i)| \equiv 1(\bmod 3)$ for any $i, j \in S$, then $f(i) \equiv i(\bmod 3)$.

Proof. The monotonicity of $f$ - and the rest of the hypotheses - provides that $f(i+1)-$ $f(i) \equiv 1(\bmod 3)$, for each $1 \leq i<n$; apply it inductively to reach the conclusion.

Theorem 4.8. Let $G=C_{3 k+2}$ for a positive integer $k$, and let $f: V\left(G_{1}\right) \rightarrow V\left(G_{2}\right)$ be a permutation, where the vertices in both the domain and codomain are labeled 1 through $3 k+2$. Assume

$$
d_{G_{2}}(f(x), f(y)) \equiv 1 \quad(\bmod 3) \text { whenever } d_{G_{1}}(x, y) \equiv 1 \quad(\bmod 3) .
$$

If $f(1)=1$, then $C\left(C_{3 k+2}, f\right) \cong C_{3 k+2} \times K_{2}$.

Proof. Denote by $F(n)$ the sequence of inequalities $f(1)<f(2)<\cdots<f(n-1)<f(n)$. By cyclically relabeling (equivalent to going to an isomorphic graph) if necessary, we may assume $F(3)$; now the graph $C\left(C_{3 k+2}, f\right)$, along with the labeling of all its vertices, is fixed. Without loss of generality, let $f(1)=1, f(2)=3 y_{0}+2$, and $f(3)=3 z_{0}+3$ for $0 \leq y_{0} \leq z_{0}<k$. Notice $|x-y| \equiv 1(\bmod 3)$ if and only if $d_{G}(x, y) \equiv 1(\bmod 3)$ for $G=C_{3 k+2}$; we will use $|\cdot|$ in distance considerations. We will prove that $f$ is monotone increasing on vertices in $G_{1}$ (and hence $f$ is the identity function) in two steps: Step I is the extension to $F(5)$ from $F(3)$. Step II is the extension to $F(3(m+1)+2)$ from $F(3 m+2)$ if $1 \leq m \leq k-1$. 
Step I. Suppose for the sake of contradiction that $F(5)$ is false. We first prove $F(4)$ and then $F(5)$.

Suppose $f(4)<f(3)$. This means, by condition (1), that $f(4) \equiv 2(\bmod 3)$. If $f(5)<f(4)$, then condition (11) implies $f(5) \equiv 1(\bmod 3)$. If $f(5)>f(4)$, then condition (1D) implies $f(5) \equiv 0(\bmod 3)$. Now notice $|1-5| \equiv 1(\bmod 3)$. If $f(5)<f(4)$, then $|f(1)-f(5)|=$ $f(5)-f(1) \equiv 0(\bmod 3)$; if $f(5)>f(4)$, then $|f(1)-f(5)|=f(5)-f(1) \equiv 2(\bmod 3)$. In either case, condition (1) is violated. Thus $f(3)<f(4)$, and $f(4) \equiv 1(\bmod 3)$.

Suppose $f(5)<f(4)$. This means, by condition (1), that $f(5) \equiv 0(\bmod 3)$. Then $|f(1)-f(5)|=f(5)-f(1) \equiv 2(\bmod 3)$, which contradicts condition (11) since, again, $|1-5| \equiv 1(\bmod 3)$. Thus we have $f(4)<f(5)$, and $f(5) \equiv 2(\bmod 3)$.

Step II. Suppose $F(3 m+2)$ for $1 \leq m \leq k-1$; we will show $F(3(m+1)+2)$. Observe that

$$
f(3 m+5)-f(1) \equiv 1 \quad(\bmod 3) \text { implies } f(3 m+5) \equiv 2 \quad(\bmod 3) .
$$

First, assume $f(3 m+3)<f(3 m+2)$ : This means, by condition (11) and Lemma 4.7, that $f(3 m+3) \equiv 1(\bmod 3)$. Assuming $f(3 m+4)>f(3 m+3)$, then $f(3 m+4) \equiv 2(\bmod 3)$; which in turn implies that $f(3 m+5) \equiv 0$ or $1(\bmod 3)$, either way a contradiction to (2). Assuming $f(3 m+4)<f(3 m+3)$, then $f(3 m+4) \equiv 0(\bmod 3)$; however, comparing with $f(3), f(3 m+4) \equiv 1$ or $2(\bmod 3)$, either way a contradiction again. We have thus shown that $f(3 m+3)>f(3 m+2)$, which means $f(3 m+3) \equiv 0(\bmod 3)$.

Second, assume $f(3 m+4)<f(3 m+3)$ : This means, by condition (11) and Lemma 4.7. that $f(3 m+4) \equiv 2(\bmod 3)$. Assuming $f(3 m+5)>f(3 m+4)$, we have $f(3 m+5) \equiv 0$ $(\bmod 3)$. Assuming $f(3 m+5)<f(3 m+4)$, we have $f(3 m+5) \equiv 1(\bmod 3)$. Either way we reach a contradiction to (2) . We have thus shown that $f(3 m+4)>f(3 m+3)$, which means $f(3 m+4) \equiv 1(\bmod 3)$.

Finally, assume $f(3 m+5)<f(3 m+4)$ : This means, by condition (11) and Lemma 4.7, that $f(3 m+5) \equiv 0(\bmod 3)$, which is a contradiction to (2). Thus, $f(3 m+5)>f(3 m+4)$ and $f(3 m+5) \equiv 2(\bmod 3)$.

Theorem 4.9. For any function $f, \gamma\left(C\left(C_{3 k+2}, f\right)\right)<2 \gamma\left(C_{3 k+2}\right)$, where $k \in \mathbb{Z}^{+}$.

Proof. Combine Theorem 3.5. Theorem 4.5, Theorem 4.6, and Theorem 4.8.

\subsection{Towards a characterization of $\gamma\left(C\left(C_{3 k}, f\right)\right)$}

Definition 4.10. Let $f$ be a function from $S=\{1,2, \ldots, 3 k\}$ to itself. We say $f$ is a three-translate if $f(x+3 i)=f(x)+3 i$ for $x \in\{1,2,3\}$ and $i \in\{0,1, \ldots, k-1\}$. Let $\widetilde{f}=\left.f\right|_{\{1,2,3\}}$.

Notation. Denote by $\widetilde{f}=\left(a_{1}, a_{2}, a_{3}\right)$ the function such that $\widetilde{f}(1)=a_{1}, \tilde{f}(2)=a_{2}$, and $\widetilde{f}(3)=a_{3}$. We use $C\left(C_{3 k}, f\right)$ and $C\left(C_{3 k}, \widetilde{f}\right)$ interchangeably when $f$ is a three-translate.

First consider $C\left(C_{3 k}, f\right)$ for a three-translate permutation $f$. 


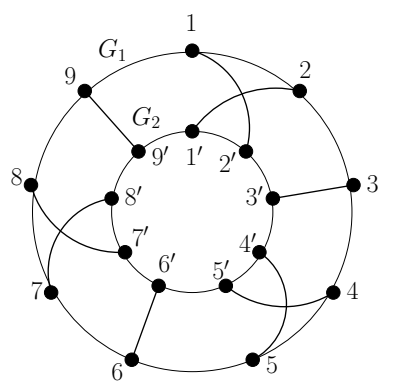

(A)

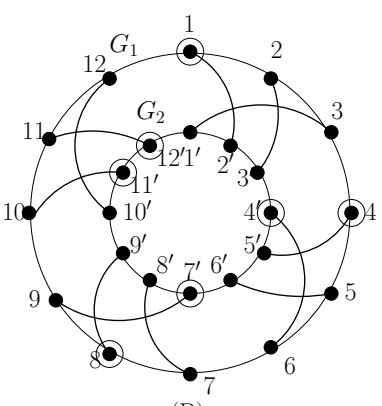

(B)

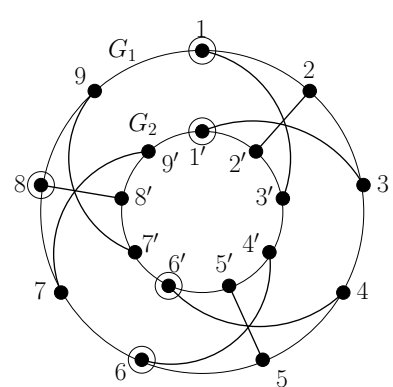

(C)

Figure 6: Examples of $C\left(C_{3 k}, f\right)$ for three-translate permutations $f$ when $k \geq 3$

Theorem 4.11. Let $f$ be a three-translate permutation and let $k \geq 4$. Then $\gamma\left(C\left(C_{3 k}, f\right)\right)=$ $2 k=2 \gamma\left(C_{3 k}\right)$ if and only if $\tilde{f}$ is $(2,1,3)$ or $(1,3,2)$.

Proof. Notice that $\tilde{f}$ is one of the six permutations: identity, $(1,3,2),(2,1,3),(2,3,1)$, $(3,1,2)$, and $(3,2,1)$. First, the identity does not attain the upper bound for $k \geq 3$ by Corollary 3.6. Second, the permutations $(2,3,1)$ and $(3,1,2)$ are inverses of each other and induce isomorphic graphs in $C\left(C_{3 k}, f\right)$; they do not attain the upper bound for $k \geq 4$ : $D=\left\{1,4,8,4^{\prime}, 7^{\prime}, 11^{\prime}, 12^{\prime}\right\}$ is a dominating set of $C\left(C_{12}, f\right)$ where $\widetilde{f}=(2,3,1)$ (see (B) of Figure 6). Third, the transposition $(3,2,1)$ fails to attain the upper bound for $k \geq 3$ : $D=\left\{1,6,8,1^{\prime}, 6^{\prime}\right\}$ is a dominating set of $C\left(C_{9}, f\right)$ (see (C) of Figure 6 ). When $\widetilde{f}$ is $(2,3,1)$ or $(3,1,2)$ or $(3,2,1)$, one can readily see how to extend a dominating set from $k$ to $k+1$. Lastly, the transpositions $(1,3,2)$ and $(2,1,3)$ induce isomorphic graphs in $C\left(C_{3 k}, f\right)$.

Claim: If $\tilde{f}$ is $(1,3,2)$ or $(2,1,3)$, then $\gamma\left(C\left(C_{3 k}, f\right)\right)=2 k=2 \gamma\left(C_{3 k}\right)$ for each $k \geq 3$.

For definiteness, let $\widetilde{f}=(2,1,3)$ (see (A) of Figure 6 ). For the sake of contradiction, assume $\gamma\left(C\left(C_{3 k}, f\right)\right)<2 \gamma\left(C_{3 k}\right)=2 k$ and consider a minimum dominating set $D$ for $C\left(C_{3 k}, f\right)$. We can partition the vertices into $k$ sets $S_{i}=\left\{u_{3 i-2}, u_{3 i-1}, u_{3 i}, v_{3 i-2}, v_{3 i-1}, v_{3 i}\right\}$ for $1 \leq i \leq k$. By the Pigeonhole Principle, $\left|D \cap S_{i}\right| \leq 1$ for some $i$. Without loss of generality, we assume that $\left|D \cap S_{1}\right| \leq 1$. Since neither $u_{2}$ nor $v_{2}$ has a neighbor that is not in $S_{1}, D \cap S_{1}$ must be either $\left\{u_{1}\right\}$ or $\left\{v_{1}\right\}$ - in order for both $u_{2}$ and $v_{2}$ to be dominated by only one vertex.

Notice that $u_{3}$ and $v_{3}$ are dominated neither by $u_{1}$ nor by $v_{1}$, so $D \cap S_{2}$ must contain both $u_{4}$ and $v_{4}$. But then either $\left|D \cap S_{2}\right| \geq 3$ or $u_{6}$ and $v_{6}$ are not dominated by any vertex in $D \cap S_{2}$ : if $\left|D \cap S_{2}\right| \geq 3$, we start the argument anew at $S_{3}$; thus we may, without loss of generality, assume $u_{6}$ and $v_{6}$ are not dominated by any vertex in $D \cap S_{2}$ and $\left|D \cap S_{2}\right|=2$. This forces $u_{7}$ and $v_{7}$ to be in $D$, but this still leaves $u_{9}$ and $v_{9}$ un-dominated by any vertex in $\cup_{i=1}^{3}\left(D \cap S_{i}\right)$. Again, if $\left|D \cap S_{3}\right| \geq 3$, we start the argument anew at $S_{4}$. Thus, we may assume $u_{9}$ and $v_{9}$ are not dominated by any vertex in $\cup_{i=1}^{3}\left(D \cap S_{i}\right)$.

This pattern (allowing restarts) is forced to persist if $\gamma\left(C\left(C_{3 k}, f\right)\right)<2 k$. Now, one of two situations prevails for $U_{k}$ : First, the argument begins anew at $U_{k}$. In this case, even if $u_{3 k-2}$ and $v_{3 k-2}$ are dominated by vertices outside $S_{k}$, one still has $\left|D \cap S_{k}\right| \geq 2$, and hence $|D| \geq 2 k$. Second, the vertices $u_{3 k-2}$ and $v_{3 k-2}$ are already in $D$. And if $\left|D \cap S_{k}\right|=2$, 
then either $u_{3 k}$ or $v_{3 k}$ is left un-dominated. Therefore, $\left|D \cap S_{k}\right| \geq 3$; this means $|D| \geq 2 k$, contradicting the original hypothesis.

Remark 4.12. One can readily check that $\gamma\left(C\left(C_{12 k},(2,3,1)\right)\right)=\gamma\left(C\left(C_{12 k},(3,1,2)\right)\right) \leq 7 k$ and $\gamma\left(C\left(C_{9 k},(3,2,1)\right)\right) \leq 5 k$ for $k \in \mathbb{Z}^{+}$.

Next we consider $C\left(C_{3 k}, f\right)$ for a non-permutation three-translate $f$. Note that constant three-translates (i.e., $\tilde{f}=$ constant) never achieve the upper bound.

Remark 4.13. For $k \geq 3$, it is easy to check that there are five non-isomorphic and non-constant three-translates which are not permutations. That is, (i) $C\left(C_{3 k},(1,1,2)\right) \cong$ $C\left(C_{3 k},(1,1,3)\right) \cong C\left(C_{3 k},(1,2,2)\right) \cong C\left(C_{3 k},(2,2,3)\right) \cong C\left(C_{3 k},(1,3,3)\right) \cong C\left(C_{3 k},(2,3,3)\right)$; (ii) $C\left(C_{3 k},(1,2,1)\right) \cong C\left(C_{3 k},(2,1,2)\right) \cong C\left(C_{3 k},(2,3,2)\right) \cong C\left(C_{3 k},(3,2,3)\right)$; (iii) $C\left(C_{3 k},(2,1,1)\right)$ $\cong C\left(C_{3 k},(2,2,1)\right) \cong C\left(C_{3 k},(3,2,2)\right) \cong C\left(C_{3 k},(3,3,2)\right)$; (iv) $C\left(C_{3 k},(1,3,1)\right) \cong C\left(C_{3 k},(3,1,3)\right)$; (v) $C\left(C_{3 k},(3,1,1)\right) \cong C\left(C_{3 k},(3,3,1)\right)$.

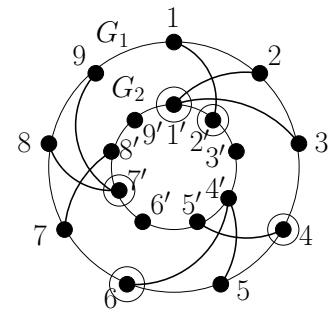

$$
D=\left\{4,6,1^{\prime}, 2^{\prime}, 7^{\prime}\right\}
$$

(A)

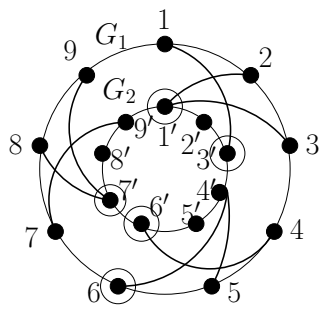

$$
D=\left\{6,1^{\prime}, 3^{\prime}, 6^{\prime}, 7^{\prime}\right\}
$$

(B)

Figure 7: Examples of $C\left(C_{3 k}, f\right)$ such that $\gamma\left(C\left(C_{3 k}, f\right)\right)<2 \gamma\left(C_{3 k}\right)$ for non-permutation three-translates $f$ and for $k \geq 3$

Theorem 4.14. Let $f$ be a three-translate which is not a permutation and let $k \geq 3$. Then $\gamma\left(C\left(C_{3 k}, \widetilde{f}\right)\right)=2 k=2 \gamma\left(C_{3 k}\right)$ if and only if $C\left(C_{3 k}, \widetilde{f}\right) \cong C\left(C_{3 k},(1,1,2)\right)$ or $C\left(C_{3 k}, \widetilde{f}\right) \cong$ $C\left(C_{3 k},(1,2,1)\right)$ or $C\left(C_{3 k}, \widetilde{f}\right) \cong C\left(C_{3 k},(1,3,1)\right)$.

Proof. There are 21 functions which are not permutations from $S=\{1,2,3\}$ to itself. The three constant functions obviously fail to achieve the upper bound (if $\widetilde{f} \equiv$ constant, then $\left.\gamma\left(C\left(C_{3 k}, \tilde{f}\right)\right)=\gamma\left(C_{3 k}\right)=k\right)$; so there are 18 non-permutation functions to consider. By Remark 4.13, we need to consider five non-isomorphic classes.

First, we consider when the domination number of $C\left(C_{3 k}, f\right)$ is less than $2 \gamma\left(C_{3 k}\right)=2 k$. If $C\left(C_{3 k}, \widetilde{f}\right) \cong C\left(C_{3 k},(2,1,1)\right)$, then $D=\left\{4,6,1^{\prime}, 2^{\prime}, 7^{\prime}\right\}$ is a dominating set of $C\left(C_{9},(2,1,1)\right)$ (see (A) of Figure 7). If $C\left(C_{3 k}, \widetilde{f}\right) \cong C\left(C_{3 k},(3,1,1)\right)$, then $D=\left\{6,1^{\prime}, 3^{\prime}, 6^{\prime}, 7^{\prime}\right\}$ is a dominating set of $C\left(C_{9},(3,1,1)\right)$ (see (B) of Figure 7). In each case, $|D|=5<2 \gamma\left(C_{9}\right)$, and one can readily see how to extend a dominating set from $k$ to $k+1$ such that $\gamma\left(C\left(C_{3 k}, \widetilde{f}\right)\right)<$ $2 \gamma\left(C_{3 k}\right)=2 k$. 
Second, we consider when $C\left(C_{3 k}, \widetilde{f}\right) \cong C\left(C_{3 k},(1,1,2)\right)$ or $C\left(C_{3 k}, \widetilde{f}\right) \cong C\left(C_{3 k},(1,2,1)\right)$ or $C\left(C_{3 k}, \widetilde{f}\right) \cong C\left(C_{3 k},(1,3,1)\right.$ ) (see Figure 8 ). In all three cases, $\gamma\left(C\left(C_{3 k}, \widetilde{f}\right)\right)=2 \gamma\left(C_{3 k}\right)$ and our proofs for the three cases agree in the main idea but differ in details.

Here is the main idea. Since one can explicitly check the few cases when $k<3$, assume $k \geq 3$. In all three cases, we view $C\left(C_{3 k}, \widetilde{f}\right)$ as the union of $k$ subgraphs $\left\langle U_{i}\right\rangle$ for $1 \leq i \leq k$, where $U_{i}=\left\{u_{3 i-2}, u_{3 i-1}, u_{3 i}, v_{3 i-2}, v_{3 i-1}, v_{3 i}\right\}$, together with two additional edges between $U_{i}$ and $U_{j}$ exactly when $i-j \equiv-1$ or $1(\bmod k)$. For each $i$, the presence of internal vertices in $U_{i}$ (vertices which can not be dominated from outside of $U_{i}$ ) imply the inequality $\left|D \cap U_{i}\right| \geq 1$. Assuming, for the sake of contradiction, that there exists a minimum dominating set $D$ with $|D|<2 k$, we conclude, by the pigeonhole principle, the existence of a "deficient $U_{p}$ " (i.e., $\left|D \cap U_{p}\right|=1<2$ ). Starting at this $U_{p}$ and sequentially going through each $U_{i}$, we can argue that this deficient $U_{p}$ is necessarily compensated (or "paired off") by an "excessive $U_{q}$ " (i.e., $\left|D \cap U_{q}\right|>2$ ). Going through all indices in $\{1,2, \ldots, k\}$, we are forced to conclude that $|D| \geq 2 k$, contradicting our hypothesis. To avoid undue repetitiveness, we provide a detailed proof only in one of the three cases, the case of $C\left(C_{3 k},(1,3,1)\right)$, which is isomorphic to $C\left(C_{3 k},(3,1,3)\right)$.

Claim: If $C\left(C_{3 k}, \widetilde{f}\right) \cong C\left(C_{3 k},(3,1,3)\right)$, then $\gamma\left(C\left(C_{3 k}, f\right)\right)=2 k=2 \gamma\left(C_{3 k}\right)$.

Proof of Claim. The assertion may be explicitly verified for $k<4$; so let $k \geq 4$. For the sake of contradiction, assume $\gamma\left(C\left(C_{3 k}, f\right)\right)<2 k$ and consider a minimum dominating set $D$ for $C\left(C_{3 k}, f\right)$. We can partition the vertices into $k$ sets $U_{i}=\left\{u_{3 i-2}, u_{3 i-1}, u_{3 i}, v_{3 i-2}, v_{3 i-1}, v_{3 i}\right\}$ for $1 \leq i \leq k$. By the Pigeonhole Principle, $\left|D \cap U_{i}\right| \leq 1$ for some $i$. Without loss of generality, we assume that $\left|D \cap U_{1}\right| \leq 1$. Since neither $u_{2}$ nor $v_{2}$ has a neighbor that is not in $U_{1}, D \cap U_{1}$ must be $\left\{v_{1}\right\}$ - the only vertex to dominate both $u_{2}$ and $v_{2}$.

Notice that $u_{3}$ and $v_{3}$ are not dominated by $v_{1}$, the only vertex in $D \cap U_{1}$, so $D \cap U_{2}$ must contain both $u_{4}$ and $v_{4}$. But then either $\left|D \cap U_{2}\right| \geq 3$ or $u_{6}$ is not dominated by any vertex in $D \cap U_{2}$ : if $\left|D \cap U_{2}\right| \geq 3$, we start the argument anew at $U_{3}$; thus we may, without loss of generality, assume $u_{6}$ is not dominated by any vertex in $D \cap U_{2}$. This forces $u_{7}$, which dominates $u_{6}, u_{8}$, and $v_{9}$, to be in $D$. Now, for $v_{7}$ and $v_{8}$ to be dominated, one of them must be in $D$. But this still leaves $u_{9}$ un-dominated by any vertex in $\cup_{i=1}^{3} U_{i}$. Again, if $\left|D \cap U_{3}\right| \geq 3$, we start the argument anew at $U_{4}$. Thus, we may, without loss of generality, assume $u_{9}$ is not dominated by any vertex in $\cup_{i=1}^{3} U_{i}$.

This pattern (allowing restarts) is forced to persist if $\gamma\left(C\left(C_{3 k}, f\right)\right)<2 k$. Now, one of two situations prevails for $U_{k}$ : First, the argument begins anew at $U_{k}$. In this case, even if $u_{3 k-2}$ and $v_{3 k-2}$ are dominated by vertices outside of $U_{k}$, one still has $\left|D \cap U_{k}\right| \geq 2$, and hence $|D| \geq 2 k$. Second, the vertices $u_{3 k-2}$ and either $v_{3 k-2}$ or $v_{3 k-1}$ are already in $D$. And if $\left|D \cap U_{k}\right|=2$, then $u_{3 k}$ (and, for that matter, $u_{1}$ ) is left un-dominated. Therefore, $\left|D \cap U_{k}\right| \geq 3$ and $|D| \geq 2 k$, contradicting the original hypothesis. 


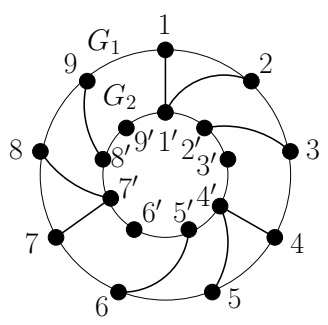

$C\left(C_{9},(1,1,2)\right)$

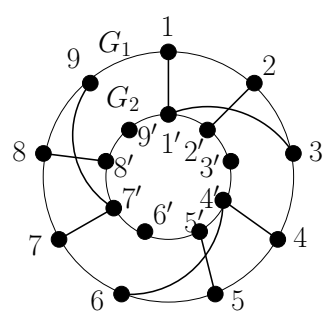

$C\left(C_{9},(1,2,1)\right)$

(B)

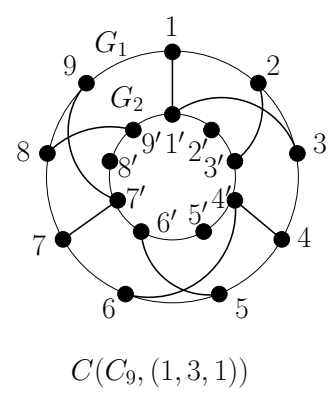

(C)

Figure 8: Examples of $C\left(C_{3 k}, f\right)$ such that $\gamma\left(C\left(C_{3 k}, f\right)\right)=2 \gamma\left(C_{3 k}\right)$ for non-permutation three-translates $f$ and for $k \geq 3$

Now, we consider sufficient conditions for $\gamma\left(C\left(C_{3 k}, f\right)\right)<2 \gamma\left(C_{3 k}\right)$ in terms of the maximum and the average degree of $C\left(C_{3 k}, f\right)$, respectively.

Proposition 4.15. If $\Delta\left(C\left(C_{3 k}, f\right)\right) \geq k+5$, then $\gamma\left(C\left(C_{3 k}, f\right)\right)<2 \gamma\left(C_{3 k}\right)$.

Proof. Suppose $C\left(C_{3 k}, f\right)$ is a functigraph with maximum degree at least $k+5$. Without loss of generality, we assume that the degree of $v_{1}$ is at least $k+5$. Partition the vertices of $G_{1}$ into $k$ sets $U_{i}=\left\{u_{3 i-2}, u_{3 i-1}, u_{3 i}\right\}$, where $1 \leq i \leq k$. If $N\left[v_{1}\right]$ contains any set $U_{i}$, say $U_{1} \subseteq N\left[v_{1}\right]$, then $\left\{u_{i} \mid i \geq 5\right.$ and $\left.i \equiv 2(\bmod 3)\right\} \cup\left\{v_{i} \mid i \equiv 1(\bmod 3)\right\}$ is a dominating set of $C\left(C_{3 k}, f\right)$ with $2 k-1$ vertices. Thus, we may assume that $\left|N\left[v_{1}\right] \cap U_{i}\right| \leq 2$ for each $i$. It follows that $\left|N\left[v_{1}\right] \cap U_{i}\right|=2$ for at least 3 different values of $i$, say $i=p, q$, and $r$. Let $x, y$, and $z$ be the vertices in $G_{1}$ that are in $U_{p}, U_{q}, U_{r}$ (respectively) and not in $N\left[v_{1}\right]$.

Suppose one of $x, y$, and $z$, say $x$, maps to a vertex $v_{3 j+1}$ for some $j$. Then $\left\{u_{\ell} \mid \ell \equiv 2\right.$ $(\bmod 3)$ and $\ell \neq 3 p-1\} \cup\left\{v_{\ell} \mid \ell \equiv 1(\bmod 3)\right\}$ is a dominating set of $C\left(C_{3 k}, f\right)$ with $2 k-1$ vertices. Otherwise, two of $x, y$, and $z$, say $x$ and $y$, map to vertices $v_{s}$ and $v_{t}$ such that $s \equiv t$ $(\bmod 3)$, say $s \equiv t \equiv 0(\bmod 3)$, without loss of generality. But then the set $\left\{u_{\ell} \mid \ell \equiv 2\right.$ $(\bmod 3), \ell \neq 3 p-1$, and $\ell \neq 3 q-1\} \cup\left\{v_{1}\right\} \cup\left\{v_{\ell} \mid \ell \equiv 0(\bmod 3)\right\}$ is a dominating set of $C\left(C_{3 k}, f\right)$ with $2 k-1$ vertices.

The following example shows that the bound provided in Proposition 4.15 is nearly sharp. Namely, there exists a function $f: V\left(C_{3 k}\right) \rightarrow V\left(C_{3 k}\right)$ such that the resulting functigraph has $\Delta\left(C\left(C_{3 k}, f\right)\right)=k+3$ and $\gamma\left(C\left(C_{3 k}, f\right)\right)=2 \gamma\left(C_{3 k}\right)=2 k$.

Example 4.16. For $k \in \mathbb{Z}^{+}$, let $f: V\left(C_{3 k}\right) \rightarrow V\left(C_{3 k}\right)$ be a function defined by

$$
f\left(u_{i}\right)=\left\{\begin{array}{lll}
v_{i} & \text { if } i \equiv 1 & (\bmod 3), \\
v_{i+1} & \text { if } i \equiv 2 & (\bmod 3), \\
v_{3 k} & \text { if } i \equiv 0 & (\bmod 3) .
\end{array}\right.
$$

Then $\gamma\left(C\left(C_{3 k}, f\right)\right)=2 k=2 \gamma\left(C_{3 k}\right)$. 
Proof. Notice that $\Delta\left(C\left(C_{3 k}, f\right)\right)=\operatorname{deg}\left(v_{3 k}\right)=k+3$. For $1 \leq i \leq k$, define $S_{i}=$ $\left\{u_{3 i}, u_{3 i-1}, u_{3 i-2}, v_{3 i}, v_{3 i-1}, v_{3 i-2}\right\}$, and notice that $\cup_{i=1}^{k} S_{i}$ is a partition of $V\left(C\left(C_{3 k}, f\right)\right)$. Let $D$ be any dominating set of $C\left(C_{3 k}, f\right)$; we need to show that $|D| \geq 2 k$. Observe that $\left|D \cap S_{i}\right| \geq 1$ since neither $u_{3 i-1}$ nor $v_{3 i-1}$ can be dominated from outside of $S_{i}$ for $1 \leq i \leq k$. We will argue in an inductive fashion starting at $k$ and descending to 1.

Suppose $|D|<2 k$; choose the biggest $j \leq k$ such that $\left|D \cap S_{j}\right|=1$. Of necessity $v_{3 j} \in D$, as it is the only vertex in $S_{j}$ dominating both $u_{3 j-1}$ and $v_{3 j-1}$. Then $\left|D \cap S_{j-1}\right| \geq 2$, since to dominate $u_{3 j-2}$ and $v_{3 j-2}$ in $S_{j}, D$ must contain both $u_{3 j-3}$ and $v_{3 j-3}$ in $S_{j-1}$.

Now, if $\left|D \cap S_{j-1}\right| \geq 3$, then it is "paired off" with $S_{j}$. We will choose the biggest $\ell<j$ such that $\left|D \cap S_{\ell}\right|=1$ and restart at $S_{\ell}$ our inductive argument. Of course, $S_{j}$ may be paired off with $S_{q}$ where $j>q \geq 1$ and $\left|D \cap S_{q}\right| \geq 3$; in this case, of necessity, $\left|D \cap S_{p}\right|=2$ for $j>p>q$, and we restart the argument after $S_{q}$ when $q>1$. Therefore, one of the following cases must hold for $S_{1}$.

(i) $\left|D \cap S_{1}\right| \geq 3$ : then $S_{1}$ may be paired off with the least $j$ such that $\left|D \cap S_{j}\right|=1$, if necessary.

(ii) $\left|D \cap S_{1}\right|=2$ and every $S_{j}$ with $\left|D \cap S_{j}\right|=1$ is paired off with $S_{q}$ such that $q<j$ and $\left|D \cap S_{q}\right| \geq 3$.

(iii) $\left|D \cap S_{1}\right|=2$ and there exists $j>1$ with $\left|D \cap S_{j}\right|=1$ which is not paired off with some $S_{q}$ such that $q<j$ and $\left|D \cap S_{q}\right| \geq 3$ : If $j=k$, then by examining $S_{k}, S_{k-1}$, and $S_{1}$, we will readily see that the assumption is impossible ( $u_{1}$ is not dominated). If $j<k$, then there must exist $q>j$ such that $\left|D \cap S_{q}\right| \geq 3$ (in order to dominate $u_{3(j+1)-2}$ ).

(iv) $\left|D \cap S_{1}\right|=1$ : then there must exist $q>1$ such that $\left|D \cap S_{q}\right| \geq 3$ (in order to dominate $u_{4}$ ).

In each case, we conclude $|D| \geq 2 k$, contradicting our original supposition.

Proposition 4.17. Suppose $C\left(C_{3 k}, f\right)$ is a functigraph with domain $G_{1}$ and codomain $G_{2}$. Partition $G_{2}$ into three sets $V_{1}, V_{2}$, and $V_{3}$ such that $V_{i}=\left\{v_{j} \mid j \equiv i(\bmod 3)\right\}$. If there is some $i$ such that the average degree over all vertices in $V_{i}$ is strictly greater than 4 , then $\gamma\left(C\left(C_{3 k}, f\right)\right)<2 \gamma\left(C_{3 k}\right)$.

Proof. Suppose $C\left(C_{3 k}, f\right)$ is a functigraph with codomain $G_{2}$ and that there is some $i$, say $i=1$, such that the average degree over all vertices in $V_{1}$ is strictly greater than 4 . Then $\left|N\left[V_{1}\right] \cap V\left(G_{1}\right)\right| \geq 2 k+1$. Let $U_{1}$ be the vertices in $V\left(G_{1}\right)$ that are not in $N\left[V_{1}\right]$ and notice that $\left|U_{1}\right| \leq k-1$. Then $U_{1} \cup V_{1}$ is a dominating set of $C\left(C_{3 k}, f\right)$.

Remark 4.18. The result obtained in Proposition 4.17 is sharp as shown in Example 4.16. In the example, the average degree of the vertices in $V_{3}$ is exactly 4.

Acknowledgement. The authors wish to thank Andrew Chen for a motivating example - the graph (B) in Figure 8. The authors also thank the referees and the editor for corrections and suggestions, which improved the paper. 


\section{References}

[1] S. Benecke, Domination of generalized Cartesian products, Ph.D. Dissertation, University of Victoria (2009).

[2] S. Benecke and C.M. Mynhardt, Domination of generalized Cartesian products, Discrete Math. 310 (2010) 1392-1397.

[3] C. Berge, Graphs and Hypergraphs (North-Holland, Amsterdam,1973).

[4] C. Berge, Theory of Graphs and its Applications (Methuen, London, 1962).

[5] A.P. Burger and C.M. Mynhardt, Regular graphs are not universal fixers, Discrete Math. 310 (2010) 364-368.

[6] A.P. Burger, C.M. Mynhardt, and W. D. Weakley, On the domination number of prisms of graphs, Discuss. Math. Graph Theory 24 (2004) 303-318.

[7] G. Chartrand and F. Harary, Planar permutation graphs, Ann. Inst. H. Poincare (Sect. B) 3 (1967) 433-438.

[8] G. Chartrand and P. Zhang, Introduction to Graph Theory (McGraw-Hill, Kalamazoo, MI, 2004).

[9] A. Chen, D. Ferrero, R. Gera, and E. Yi, Functigraphs: An Extension of Permutation Graphs, Math. Bohem. 136, No. 1 (2011) 27-37.

[10] E.J. Cockayne and S.T. Hedetniemi, Towards a Theory of Domination in Graphs, Networks 7 (1977) 247-261.

[11] W. Dörfler, On mapping graphs and permutation graphs, Math. Solvaca 28, No. 3 (1978) 277-288.

[12] B.L. Hartnell and D.F. Rall, On dominating the cartesian product of a graph and $K_{2}$, Discuss. Math. Graph Theory 24 (2004) 389-402.

[13] T.W. Haynes, S.T. Hedetniemi, and P.J. Slater, Domination in Graphs: Advanced Topics (Marcel Dekker, New York, 1998).

[14] T.W. Haynes, S.T. Hedetniemi, and P.J. Slater, Fundamentals of Domination in Graphs (Marcel Dekker, New York, 1998).

[15] S. T. Hedetniemi, On classes of graphs defined by special cutsets of lines, Many Facets of Graph Theory, Lect. Notes Math. 110 (1969) 171-189.

[16] O. Ore, Theory of Graphs (Amer. Math. Soc. Colloq. Publ., 38, Providence, 1962). 